Antonios Bisbas, Technological Educational Institute of West Macedonia, School of Technological Applications, General Sciences Department, Kila 50100 Kozani, Greece. email: bisbas@kozani.teikoz.gr

Jörg Neunhäuserer, ${ }^{*}$ TU Clausthal, Department of Mathematics, Erzstraße 1, 38678 Clausthal-Zellerfeld Germany. email: neunchen@aol.com

\title{
ON INHOMOGENEOUS BERNOULLI CONVOLUTIONS AND RANDOM POWER SERIES
}

\begin{abstract}
We extend the results of Peres and Solomyak on absolute continuity and singularity of homogeneous Bernoulli convolutions to inhomogeneous ones and generalize the result to random power series given by inhomogeneous Markov chains. In addition we prove an Erdös-Salem type theorem for inhomogeneous Bernoulli convolutions.
\end{abstract}

\section{Introduction.}

There exists an extended literature on the fascinating field of Bernoulli convolution, see especially [9] and references in there. Two highlights in the field are the relation of Bernoulli convolutions to algebraic number theory found by Erdös [3] and Salem [13] and the proof of absolute continuity of almost all Bernoulli convolutions by Solomyak [15], which was simplified and generalized to biased convolutions by Peres and Solomyak, see [10] and [11]. In this paper we ask the natural question if these results may be generalized to inhomogeneous Bernoulli convolutions having different probabilities of the convolved

\footnotetext{
Mathematical Reviews subject classification: Primary: 26A46, 26A30; Secondary: 28A80, 28A78, 11R06

Key words: inhomogeneous Bernoulli convolution, random power series, absolute continuity, singularity, Pisot numbers

Received by the editors January 28, 2010

Communicated by: Zoltn Buczolich

* Supported by the Marie Curie Training Network ,"Conformal Structures and Dynamics" of the European Community
} 
measures. We will show that this is in fact true. We will prove an analogue of the Peres and Solomyak theorem and analogue of the Erdös-Salem theorem in the inhomogeneous setting. We need some notation to state our results. For a sequence $\left(p_{n}\right)_{n \in \mathbb{N}_{0}}$ of probabilities $p_{n} \in(0,1)$ and $\beta \in(0,1)$ consider the measure $\mu_{\beta}$ given by the convolution

$$
\mu_{\beta}=*_{n=0}^{\infty}\left(p_{n} \delta_{\beta^{n}}+\left(1-p_{n}\right) \delta_{-\beta^{n}}\right),
$$

where $\delta$ is the Dirac measure. If $\left(p_{n}\right)_{n \in \mathbb{N}_{0}}$ is constant this is the classical Bernoulli convolution, see [9] and references therein. It follows from the theory of convolutions that $\mu_{\beta}$ is a Borel probability measure of pure type, either absolutely continuous or totally singular, see [4]. There are two other ways to describe the measures $\mu_{\beta}$. Consider independent real random variables $X_{n}$ with $P\left(X_{n}=1\right)=p_{n}$ and $P\left(X_{n}=-1\right)=1-p_{n}$ and the geometric series

$$
X=\sum_{n=0}^{\infty} X_{n} \beta^{n}
$$

for $\beta \in(0,1)$. Obviously $\mu_{\beta}$ is the distribution of this variable, i.e. $\mu_{\beta}(B)=$ $P(X \in B)$ for all Borel sets $B$. One may also describe $\mu_{\beta}$ as a projection of a Borel probability measure on the sequence space $\Sigma=\{-1,1\}^{\mathbb{N}_{0}}$. The sequence $\mathfrak{p}$ of probabilities induces a Borel probability measure $\mu$ on $\Sigma$ by

$$
\mu\left(\left[s_{0} \ldots s_{n}\right]\right)=\prod_{k=0}^{n}\left(\left(p_{k}-1 / 2\right) s_{k}+1 / 2\right)
$$

for a cylinder sets $\left[s_{0} \ldots s_{n}\right]$. Now $\mu_{\beta}$ is given by

$$
\mu_{\beta}=\pi(\mu)=\mu \circ \pi^{-1},
$$

where the geometric projection $\pi: \Sigma \longmapsto \mathbb{R}$ is

$$
\pi\left(\left(s_{n}\right)\right)=\sum_{n=0}^{\infty} s_{n} \beta^{n}
$$

For further use we introduce here the entropy $h(\mu)$ of an inhomogeneous Bernoulli measure $\mu$ by considering the limit of mean values,

$$
h(\mu)=\liminf _{n \longmapsto \infty}-\frac{1}{n} \sum_{i=0}^{n-1}\left(p_{i} \log \left(p_{i}\right)+\left(1-p_{i}\right) \log \left(1-p_{i}\right)\right)
$$

compare [1]. We are now prepared to state our first result. 
Theorem 1.1. For a sequence $\left(p_{n}\right)_{n \in \mathbb{N}_{0}}$ of probabilities $p_{n} \in(0,1)$ the convolution $\mu_{\beta}$ is absolutely continuous for almost all $\beta \in\left[e^{-h(\mu)}, 0.649\right]$ and singular if $\beta<e^{-h(\mu)}$. If $p_{n} \in[1 / 3,2 / 3]$ we may replace the upper bound 0.649 by 1 .

For ergodic measures $\mu$ on the sequences space a similar result was proved by Peres and Solomyak, see Theorem 4.1 of [11]. We consider here inhomogeneous Bernoulli convolutions which are in general not ergodic. The upper bound 0.649 in our result is given by an interval of transversality with respect to the coding map $\pi$, see section five of [11], and the extension of the interval is proved considering Fourier transforms and again a transversality argument. With some numerical calculations the upper bound of transversality can be extended to 0.668 , see [14]. We conjecture here that the bound can be replaced by one without an additional assumption on $p_{n}$.

Furthermore we like to remark that a result of Mauldin and Simon [8] implies the following theorem.

Theorem 1.2. Either the measure $\mu_{\beta}$ is singular or equivalent to the Lebesgue measure restricted to $[-\beta /(1-\beta), \beta /(1-\beta)]$.

We state this result without proof. It follows directly from theorem 2 of [8], since inhomogeneous Bernoulli measures $\mu$ fulfill the condition

$$
\mu(B)>0 \Rightarrow \mu((i, B))>0
$$

for $i \in\{-1,1\}$ and all Borel sets $B \subseteq \Sigma$, where $(i, B)=\{(i, j) \in \Sigma \mid j \in B\}$. Compare with condition (1) of [8].

Our next result is an analogue of classical Erdös-Salem theorem, see [3] and [13], on the relation of Bernoulli convolutions to algebraic number theory in the non homogeneous setting.

Theorem 1.3. Fix a sequence $\left(p_{n}\right)_{n \in \mathbb{N}_{0}}$ of probabilities $p_{n} \in(c, 1-c)$ for some constant $c \in(0,0.5)$. The Fourier transform $\hat{\mu}_{\beta}(\xi)$ of the convolution measure $\mu_{\beta}$ does not tend to zero for $\xi \longmapsto \infty$ if and only if $\beta$ is the reciprocal of a Pisot number.

By Riemann-Lebesgue lemma this theorem has the following Corollary:

Corollary 1.1. $\mu_{\beta}$ is singular if $\beta$ is the reciprocal of a Pisot number.

For $(1 / 2)_{n \in \mathbb{N}_{0}}$ this corollary is Erdös result [3] and for constant sequences $(p)_{n \in \mathbb{N}_{0}}$ with $p \in(0,1)$ the result was proved by Lalley $[6]$. 
Our next question is if results on convolutions may be generalized to stochastic power series given by a Markov chain. We will show that this is at least true for the results on absolute continuity and singularity of Peres and Solomyak. To this end consider a Markov chain on $\Sigma$ given by a sequence of matrices $P^{(n)}=\left(p_{i j}^{(n)}\right)_{(i, j) \in\{-1,1\}}$ for $n \in \mathbb{N}_{0}$. Here $p_{i j}^{(n)} \in(0,1)$ are the one step transition probabilities and $P^{(0)}=\left(p_{1}^{(0)}, p_{-1}^{0}\right)$ is an initial probability vector. The measure of a cylinder set in $\Sigma$ is given by

$$
\nu\left(\left[s_{0} \ldots s_{n}\right]\right)=p_{s_{0}}^{(0)} \prod_{k=0}^{n-1} p_{s_{k} s_{k+1}}^{(k)} .
$$

The entropy of the Markov measure is

$$
h(\nu)=\liminf _{n \longmapsto \infty}-\frac{1}{n} \sum_{k=0}^{n-1} \sum_{(i, j) \in\{-1,1\}} C(i, k) p_{i j}^{(k)} \log p_{i j}^{(k)}
$$

where $C(1, n)=\bar{P}^{(n)}(1,0)^{T}$ and $C(-1, n)=\bar{P}^{n}(0,1)^{T}$ with $\bar{P}^{(n)}$ defined as $\bar{P}^{(n)}=P^{(0)} P^{(1)} \cdots P^{(n-1)}$, compare [2]. By the geometric projection $\pi$ the Markov measure $\mu$ induces a Borel probability measure

$$
\nu_{\beta}=\pi(\nu)=\nu \circ \pi^{-1}
$$

on the real line. This is the distribution of the random power series

$$
Y=\sum_{n=0}^{\infty} Y_{n} \beta^{n}
$$

where $Y_{n}$ is the Markov process given by $\mu$. Theorem 1.1 has an analogue for the distribution $\nu_{\beta}$ of inhomogeneous Markov geometric series:

Theorem 1.4. Let $P^{(n)}=\left(p_{i j}^{(n)}\right)_{(i, j) \in\{-1,1\}}$ for $n \in \mathbb{N}_{0}$ with $p_{i j}^{n} \in(0,1)$ be an inhomogeneous Markov chain. The distribution $\nu_{\beta}$ of the corresponding random power series is absolutely continuous for almost all $\beta \in\left[e^{-h(\nu)}, 0.649\right]$ and singular if $\beta<e^{-h(\nu)}$.

We remark here than Fan and Zhang [5] have a result on the absolute continuity of the random power series given by the homogeneous golden Markov chain. Due to the transversality properties with respect to the coding map in this special case, their upper bound is slightly larger than our upper bound. In general it seems not to be possible to extend this bound using Fourier transform techniques. 
The rest of the paper is organized as follows. In the next section we prove our results on absolute continuity of Bernoulli convolutions $\mu_{\beta}$ and generalize them to the distribution $\nu_{\beta}$ of Markov geometric series. In section three we prove the results on singularity of these measures. The last section contains the proof of the Erdös-Salem theorem for inhomogeneous Bernoulli measures $\mu_{\beta}$.

\section{Absolute continuity of $\mu_{\beta}$ and $\nu_{\beta}$.}

For $q \in(1,2]$ let

$$
\begin{aligned}
\lambda_{q} & =\liminf _{n \longmapsto \infty} \frac{1}{n(1-q)} \log \sum_{\left(s_{0}, \ldots, s_{n-1}\right) \in\{-1,1\}^{n}}\left(\prod_{k=0}^{n-1}\left(\left(p_{k}-1 / 2\right) s_{k}+1 / 2\right)\right)^{q} \\
& =\liminf _{n \longmapsto \infty} \frac{1}{n(1-q)} \sum_{k=0}^{n-1} \log \left(p_{k}^{q}+\left(1-p_{k}\right)^{q}\right) .
\end{aligned}
$$

By part (a) of Theorem 4.1 of [11] we have that $\mu_{\beta}$ is absolutely continuous with density in $L^{q}$ for almost all $\beta \in\left[e^{-\lambda_{q}}, 0.649\right]$, since $\lambda_{q}$ is bound $D_{q}(\mu)$ defined there. Now we consider $\lambda_{q}$ for $q \longmapsto 1$. Let

$$
f(q, p)=\left\{\begin{array}{cc}
\frac{1}{1-q} \log \left(p^{q}+(1-p)^{q}\right) & \text { for } q \neq 1 \\
-(p \log (p)+(1-p) \log (1-p)) & \text { for } q=1
\end{array}\right\}
$$

By the rule of L'Hospital the function is continuous on the square $[0,1]^{2}$ and hence uniformly continuous. It follows that the convergence of mean values

$\lim _{q \longmapsto 1} \frac{1}{n(1-q)} \sum_{k=0}^{n-1} \log \left(p_{k}^{q}+\left(1-p_{k}\right)^{q}\right)=-\frac{1}{n} \sum_{k=0}^{n-1}\left(p_{k} \log \left(p_{k}\right)+\left(1-p_{k}\right) \log \left(1-p_{k}\right)\right)$

is uniform with respect to $n$. Hence

$$
\begin{aligned}
\lim _{q \longmapsto 1} \lambda_{q} & =\lim _{q \longmapsto 1} \liminf _{n \longmapsto \infty} \frac{1}{n(1-q)} \sum_{k=0}^{n-1} \log \left(p_{k}^{q}+\left(1-p_{k}\right)^{q}\right) \\
& =\liminf _{n \longmapsto \infty} \lim _{q \longmapsto 1} \frac{1}{n(1-q)} \sum_{k=0}^{n-1} \log \left(p_{k}^{q}+\left(1-p_{k}\right)^{q}\right) \\
& =\liminf _{n \longmapsto \infty}-\frac{1}{n} \sum_{k=0}^{n-1}\left(p_{k} \log \left(p_{k}\right)+\left(1-p_{k}\right) \log \left(1-p_{k}\right)\right)=h(\mu)
\end{aligned}
$$


which concludes the proof of absolute continuity in the interval $\left[e^{-h(\mu)}, 0.649\right]$.

We assume now that $p_{n} \in[1 / 3,2 / 3]$ and use the Fourier transform of $\mu_{\beta}$. The Fourier transform of a convolution is a product of Fourier transforms of the convolved measures, therefore

$\hat{\mu}_{\beta}(\xi)=\prod_{n=0}^{\infty}\left(p_{n} e^{i \beta^{n} \xi}+\left(1-p_{n}\right) e^{-i \beta^{n} \xi}\right)=\prod_{n=0}^{\infty}\left(\cos \left(\beta^{n} \xi\right)+\left(1-2 p_{n}\right) \sin \left(-\beta^{n} \xi\right) i\right)$.

Hence we have

$$
\begin{aligned}
\left|\hat{\mu}_{\beta}(\xi)\right|^{2} & =\prod_{n=0}^{\infty}\left(\cos ^{2}\left(\beta^{n} \xi\right)+\left(1-2 p_{n}\right)^{2} \sin ^{2}\left(\beta^{n} \xi\right)\right) \\
& \leq \prod_{n=0}^{\infty}\left(\cos ^{2}\left(\beta^{n} \xi\right)+\frac{1}{9} \sin ^{2}\left(\beta^{n} \xi\right)\right) .
\end{aligned}
$$

Now the right hand side is the square Fourier transform $\left|\vartheta_{\beta}\right|^{2}$ of the homogeneous Bernoulli convolution $\vartheta_{\beta}$ with $p_{n}=1 / 3$. By Corollary 1.4 of [11] $\vartheta_{\beta}$, and hence $\mu_{\beta}$, is absolutely continuous with density in $L^{2}$ for almost all $\beta \in(5 / 9,1)$. Since $5 / 9<0.649$ this concludes our argument.

It remains to establish absolute continuity of $\nu_{\beta}$ for a Markov measure $\nu$ under the assumption of theorem 1.4. Let

$$
\bar{\lambda}_{q}=\liminf _{n \longmapsto \infty} \frac{1}{n(1-q)} \log \sum_{\left(s_{0}, \ldots, s_{n-1}\right) \in\{-1,1\}^{n}}\left(p_{s_{1}}^{(0)} \prod_{k=0}^{n-1} p_{s_{k-1} s_{k}}^{(k)}\right)^{q} .
$$

Again by by part (a) of Theorem 4.1 of [11] the measure $\nu_{\beta}$ is absolutely continuous with density in $L^{q}$ for almost all $\beta \in\left[e^{-\bar{\lambda}_{q}}, 0.649\right]$. Now consider $q \longmapsto 1$. By considerations described in the case of Bernoulli measures we see that this limit is uniform with respect to $n$ and we can exchange limits. Hence using the Rule of L'Hospital

$$
\begin{aligned}
& \lim _{q \longmapsto \infty} \bar{\lambda}_{q}=\liminf _{n \longmapsto \infty} \lim _{q \longmapsto 1} \frac{1}{n(1-q)} \log \sum_{\left(s_{0}, \ldots, s_{n-1}\right) \in\{-1,1\}^{n}}\left(p_{s_{1}}^{(0)} \prod_{k=0}^{n-1} p_{s_{k-1} s_{k}}^{(k)}\right)^{q} \\
= & \liminf _{n \longmapsto \infty}-\frac{1}{n} \sum_{\left(s_{0}, \ldots, s_{n-1}\right) \in\{-1,1\}^{n}} p_{s_{1}}^{(0)} \prod_{k=0}^{n-1} p_{s_{k-1} s_{k}}^{(k)} \log \left(p_{s_{1}}^{(0)} \prod_{k=0}^{n-1} p_{s_{k-1} s_{k}}^{(k)}\right)=h(\nu),
\end{aligned}
$$

which completes the proof. 


\section{Proof of singularity of $\mu_{\beta}$ and $\nu_{\beta}$.}

Consider the space of sequence $\Sigma=\{-1,1\}^{\mathbb{N}_{0}}$ with the metric $d$ given by

$$
d(s, t)=\beta^{-|s \wedge t|}=\beta^{-\min \left\{k \mid s_{k} \neq t_{k}\right\}} .
$$

By Bisbas and Karanikas [1] we have Shannon's local entropy theorem for a non-uniform Bernoulli measure $\mu$ on $\Sigma$, that is

$$
\liminf _{n \longmapsto \infty}-\frac{1}{n} \log \mu\left(\left[s_{0}, \ldots, s_{n}\right]\right)=h(\mu)
$$

for almost all $s=\left(s_{k}\right)$. Hence we have for almost all $s \in \Sigma$

$$
\liminf _{n \longmapsto \infty} \frac{\log \mu\left(B_{\beta^{n}}(s)\right)}{\log \beta^{n}}=\frac{h(\mu)}{-\log \beta}
$$

where $B_{\beta^{n}}(s)$ is a ball with respect to the metric $d$. By Frostmann's lemma, see [12], this implies implies $\operatorname{dim}_{H} \mu=-h(\mu) / \log (\beta)$. The Hausdorff dimension here is defined with respect to the metric $d$. Now the coding map $\pi$ is Lipschitz with respect to $d$ and does hence not increase Hausdorff dimension. Consequently

$$
\operatorname{dim}_{H} \mu_{\beta}=\operatorname{dim}_{H} \pi(\mu) \leq \operatorname{dim}_{H} \mu=-h(\mu) / \log (\beta) .
$$

If $\beta<e^{-h(\mu)}$ we get $\operatorname{dim}_{H} \mu_{\beta}<1$ and $\mu_{\beta}$ is singular. Shannon's local entropy theorem remains true for non-uniform Markov measure $\nu$ on $\{-1,1\}^{\mathbb{N}}$. This fact is implicitly contained in the proof of theorem 1 of [2]. Hence by the argument described before we get

$$
\operatorname{dim}_{H} \nu_{\beta} \leq-h(\nu) / \log (\beta)
$$

and $\nu_{\beta}$ is singular if $\beta<e^{-h(\nu)}$.

\section{The Erdös-Salem type theorem.}

We prove here the Erdös-Salem type theorem for inhomogeneous Bernoulli measures $\mu_{\beta}$, see theorem 1.3.

By equation (1) of section four we get

$$
\left|\hat{\mu}_{\beta}(\xi)\right|^{2}=\prod_{n=0}^{\infty}\left(\cos ^{2}\left(\beta^{n} \xi\right)+\left(1-2 p_{n}\right)^{2} \sin ^{2}\left(-\beta^{n} \xi\right) \geq \prod_{n=0}^{\infty} \cos ^{2}\left(\beta^{n} \xi\right) .\right.
$$


for all $\xi \in \mathbb{R}$. With $\xi_{k}=2 \pi \beta^{-k}$ we get

$$
\left|\hat{\mu}_{\beta}\left(\xi_{k}\right)\right| \geq \hat{c} \prod_{n=0}^{k} \cos \left(2 \pi \beta^{-n}\right)
$$

where $\hat{c}$ is independent of $k$. Now assume that $\beta$ is the reciprocal of a Pisot number, then there is a constant $\theta \in(0,1)$ such that

$$
\min \left\{\left|\beta^{-n}-u\right| u \in \mathbb{Z}\right\}<\theta^{n} \quad \forall n \geq 0 .
$$

Hence $\left|\hat{\mu}_{\beta}\left(\xi_{k}\right)\right|$ remains bounded from below and does not tend to zero for $k \longmapsto \infty$.

Now assume that $\left|\hat{\mu}_{\beta}(\xi)\right|$ tends to zero with $\xi \longmapsto \infty$. We have

$$
\begin{aligned}
\left|\hat{\mu}_{\beta}(\xi)\right|^{2} & =\prod_{n=0}^{\infty}\left(\left(1-2 p_{n}\right)^{2} \sin ^{2}\left(\beta^{n} \xi\right)+\cos ^{2}\left(\beta^{n} \xi\right)\right) \\
& =\prod_{n=0}^{\infty}\left[1-\left(4 p_{n}-4 p_{n}^{2}\right) \sin ^{2}\left(\beta^{n} \xi\right)\right]
\end{aligned}
$$

By our assumption there is a constant $C>0$ and an increasing sequence $\xi_{s}$ such that $\left|\hat{\mu}_{\beta}\left(\pi \xi_{s}\right)\right| \geq C>0$. Writing $\xi_{s}=\lambda_{s} \beta^{-n_{s}}$ with $1 \leq \lambda_{s}<\beta^{-1}$ and $n_{s} \longmapsto \infty$ we obtain

$$
\prod_{n=0}^{n_{s}}\left[1-\left(4 p_{n}-4 p_{n}^{2}\right) \sin ^{2}\left(\pi \lambda_{s} \beta^{-n}\right)\right] \geq C^{2} .
$$

Since $1+x<e^{x}$, this implies

$$
\sum_{n=0}^{n_{s}}\left[\left(4 p_{n}-4 p_{n}^{2}\right) \sin ^{2}\left(\pi \lambda_{s} \beta^{-n}\right)\right] \leq \log \left(1 / C^{2}\right) .
$$

By choosing a subsequence we may assume that $\lambda_{s}$ converges to $\lambda \in\left[1, \beta^{-1}\right)$. Since $s$ is arbitrary large we get

$$
\sum_{n=0}^{\infty}\left[\left(4 p_{n}-4 p_{n}^{2}\right) \sin ^{2}\left(\pi \lambda \beta^{-n}\right)\right] \leq \log \left(1 / C^{2}\right) .
$$

By our assumption on $p_{n}$ we have $4 p_{n}-4 p_{n}^{2}>\bar{c}>0$. Hence

$$
\sum_{n=0}^{\infty} \sin ^{2}\left(\pi \lambda \beta^{-n}\right) \leq \bar{c}^{-1} \log \left(1 / C^{2}\right) .
$$

Now chapter I of [13] implies that $\beta$ is the reciprocal of a Pisot number. This completes the proof of theorem 1.3. 


\section{References}

[1] A. Bisbas and C. Karanikas, On the Hausdorff dimension of Rademacher Riesz products, Monatshefte für Math. 110 (1990), 15-21.

[2] A. Bisbas and C. Karanikas, Dimension and entropy of non-ergodic Markovian process and its relation to Rademacher Riez products, Monatshefte für Math. 118 (1994), 21-32.

[3] P. Erdös, On a family of symmetric Bernoulli convolutions, Amer. Journ. Math 61 (1939), 974-976.

[4] C. Graham and O.C. McGehee, Essays in commutative harmonic analysis, Fundamental principles in Mathematical Science, Springer New York - Berlin (1979).

[5] A. Fan and J. Zhang, Absolute continuity of the distribution of some Markov geometric series, Science in China. Series A. Mathematic, 50(11) (2007), 1521-1528.

[6] S. Lalley, Random series in powers of algebraic integers: Hausdorff dimension of the limit distribution, Journal of the London Mathematical Society, 57 (1998), 629-654.

[7] P. Mattila, Geometry of Sets and Measures in Euclidean spaces, Cambridge University Press (1995).

[8] D. Mauldin and K. Simon, The equivalence of some Bernoulli convolution to Lebesgue measure, Proc. of the Amer. Math. Soc. 126(9) (1998), 27332736 .

[9] Y. Peres, W. Schlag and B. Solomyak Sixty years of Bernoulli convolutions, Progress in probability 46 (2000), 39-65.

[10] Y. Peres and B. Solomyak, Absolutely continuous Bernoulli convolutions - a simple proof, Math. Research Letters 3(2) (1996), 231-239.

[11] Y. Peres and B. Solomyak, Self-similar measures and intersection of Cantor sets, Trans. Amer. Math. Soc 350(10) (1998), 4065-4087.

[12] Ya. Pesin, Dimension Theory in Dynamical Systems - Contemplary Views and Applications, University of Chicago Press (1997).

[13] R. Salem, Algebraic numbers and Fourier Analysis, Heath (1963). 
[14] P. Shmerkin and B. Solomyak, Zeros of $\{-1,0,1\}$ power series and connectedness loci of self-affine sets, Experimental Math. 15(4) (2006), 499511.

[15] B. Solomyak, On the random series $\sum \pm \lambda^{i}$ (an Erdös problem), Ann. Math. 142 (1995). 